Flow Report No. F 21-001

\title{
Performance of Non-Contact Flow Meters in Irrigation Canals and Surface Drains
}

\author{
Kyle Feist, MS, PE, M. ASCE ${ }^{1}$ \\ Jack Evans ${ }^{2}$, \\ Zack Markow, EIT $^{3}$ \\ Stuart Styles, D. Eng, PE, M. ASCE ${ }^{4}$
}

\begin{abstract}
Flow measurement is an important component of modern irrigation delivery systems. As irrigation projects are modernized, flow measurement devices are being installed in more nontraditional applications. Some applications feature a combination of characteristics that eliminate traditional flow measurement structures. Furthermore, as regulations obligate more districts to report real time diversions, redundant flow measurement devices using different methods or technologies become necessary to reduce the potential for temporary gaps in diversion data. In these cases, non-contact type flow measurement devices may be of interest. Two non-contact flow meters were installed at different irrigation district canals. Data was collected from the non-contact meters as well as a pre-existing flow measurement device operating in parallel. In addition, intermittent current metering was conducted at each site and used as the standard for calibration purposes. This paper presents an analysis of the flow measurement data collected and a discussion of the non-contact meter performance in open channel irrigation system applications.
\end{abstract}

\section{BACKGROUND}

Flow measurement is being expanded as irrigation districts modernize. Many of the new flow measurement sites lack key hydraulic characteristics necessary for traditional flow measurement structures, such as about 0.3 feet to 0.5 feet of head drop available. In addition, other new flow measurement sites are surface drains that are managed at the lowest possible water depth, as well as subjected to ephemeral flows and extreme sedimentation. For most of these applications, recent implementations have been fitted with acoustic Doppler velocity meters (ADVM).

While ADVM solutions have been relatively successful in field, various disadvantages exist. While not marketed as such, it is best practice to assume that all installations require field verification and/or calibration to provide accurate readings. ADVM readings are also prone to signal noise that can only be mitigated through substantial averaging, which is problematic for real-time automatic flow control applications. Side-mounted ADVMs will not work when water depths become extremely shallow. And lastly, bottom-mounted ADVMs are difficult to maintain with sediment and gravel-laden surface drain applications. These disadvantages leave irrigation engineers looking for alternatives.

\footnotetext{
${ }^{1}$ Senior Engineer, Irrigation Training and Research Center (ITRC), California Polytechnic State University, 1 Grand Ave, Bldg. 8A, San Luis Obispo, CA 93407-0730; e-mail: kfeist@calpoly.edu

${ }^{2}$ Support Engineer, ITRC, jevans21@calpoly.edu

${ }^{3}$ Irrigation Engineer, ITRC, zmarkow@calpoly.edu

${ }^{4}$ Director, ITRC, sstyles@calpoly.edu
} 
A new category of commercial flow measurement devices for permanent monitoring applications have been developed. This new "non-contact" flow measurement device category includes several different models, manufacturers and technologies. An incomplete list of various products in this category are listed in Table 1.

Table 1. Non-contact flow meters

\begin{tabular}{|l|l|l|l|}
\hline Manufacturer & \multicolumn{1}{|c|}{ Model(s) } & \multicolumn{1}{|c|}{ Technology } & SCADA integration? \\
\hline Hach & FloDar & Ultrasonic & Yes, via MODBUS \\
\cline { 1 - 3 } Sommer & RQ-30; SQ-30 & Radar, or ultrasonic depending on model & \\
\hline Teledyne ISCO & LaserFlow & Laser & No \\
\hline
\end{tabular}

In general, all of these devices are mounted above the water surface and use beams of electromagnetic energy (ultrasonic, radar and visible light such as lasers) to sample the surface or near-surface water velocity. A second and typically integrated sensor measures the distance to the water surface. The measured (near) surface water velocity is internally translated into an average cross-sectional velocity, coupled with the cross-sectional area computation to estimate open channel flow rates.

\section{FIELD STUDY OVERVIEW}

Non-contact meters were installed at several irrigation districts in California. Continuous data collection was provided with SCADA, remote telemetry systems or local data logging. For verification, ITRC staff visited the sites multiple times to conduct stationary current metering with a SonTek M9 ADVM boat. The SonTek M9 measurements were used as a standard in this study.

\section{Stationary M9 Current Metering Protocol}

The authors used an SonTek M9 RiverSurveyor with the Stationary Live program for standard flow measurements.

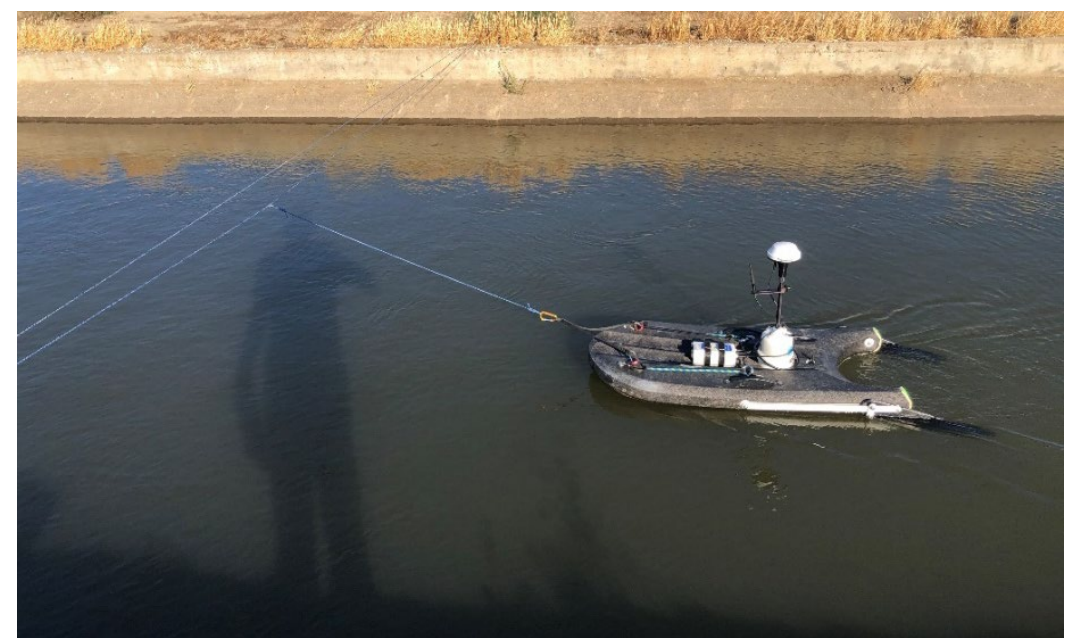

Figure 1. Configuration of the SonTek RiverSurveyor M9 for the PID main canal discharge measurement 
The stationary method divides the width of the irrigation canal into sections (stations) and uses acoustic doppler profiling (ADP) to measure the velocity at most depths at each station. The M9 has a depth range of 0.3 to 40 meters. The blanking distance (minimum depth for performing measurements) is $30 \mathrm{~cm}$ for the SonTek M9. Measurements can be performed with the survey boat on a tagline extending either from a fixed platform or by using a pulley system spanning the width of the river/canal.

\section{Stationary Method}

The stationary method involves moving the M9 ADP in increments across the canal and pausing to collect data at each increment ("station"). The authors follow USGS protocol and collects data at a minimum of 25 stations along the cross section of the canal. At each station measurements are collected for 40 seconds and averaged. The Stationary Live program uses the average water velocity, measured depth and the station width to calculate the discharge for each station. The total discharge is computed as the sum of each station's discharge.

The following additional standards were used while performing discharge measurements:

- The percent discharge of each station should be below $5 \%$ and never greater than $10 \%$ of the total discharge.

- The channel shall have a straight alignment and free of physical obstructions of its nominal cross section for at least four canal widths upstream and downstream of the measurement station.

Field Device Accuracy. The accuracy of field flow measurement devices is quantified for this study as the percent difference between the field device measurement and the SonTek M9 RiverSurveyor measurement. The following equation is used to calculate the percent difference:

$$
\text { Percent Difference }=\frac{\text { Field Device Measurement }(C F S)-\text { SonTek M9 Measurement }(C F S)}{\text { SonTek M9 Measurement }(C F S)} \times 100 \%
$$

\section{Installation \#1 Overview}

An ITRC-designed subcritical contraction is located about 180 feet downstream of a lift pump station discharge at the head of an irrigation district's pumped diversion. The site is equipped with a pre-existing SonTek SW (mounted on the structure invert) ADFM for flow measurement. A Sommers RQ30A was installed on the structure walkway. The SonTek M9 RiverSurveyor stationary discharge measurement was performed upstream of the structure. The following figure shows the configuration of the two sensors on the structure. 


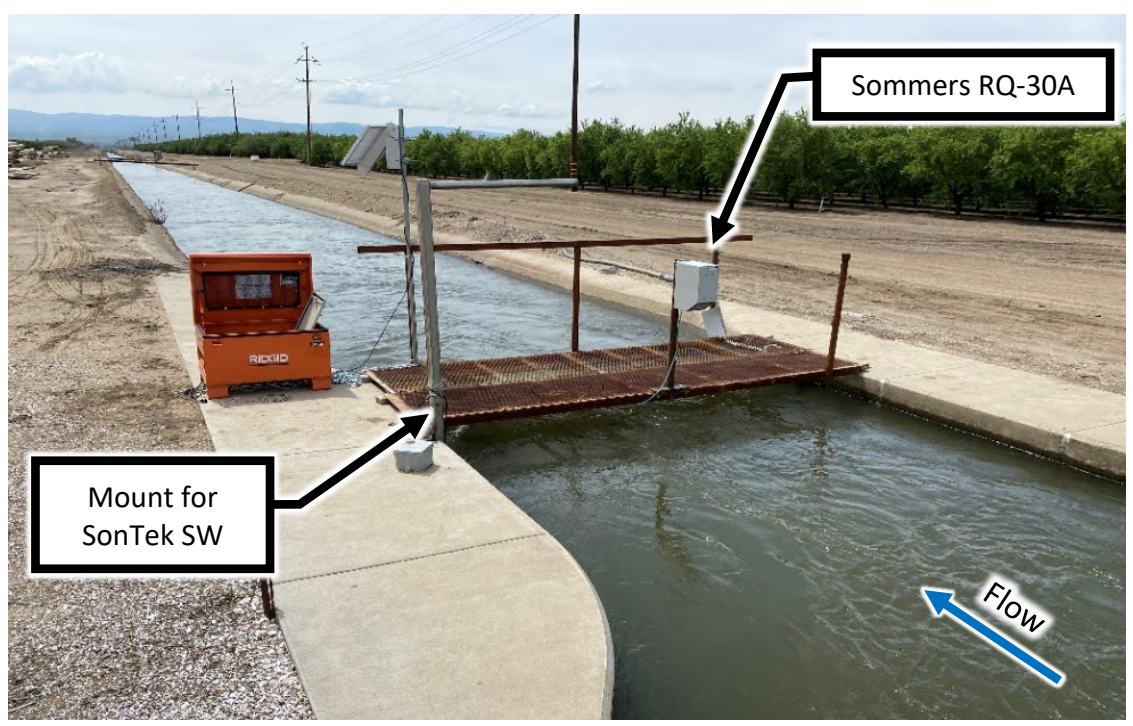

Figure 2. Installation \#1 layout

The SonTek SW is mounted to the bottom of an L-shaped stainless-steel pole and positioned in the center of the channel.

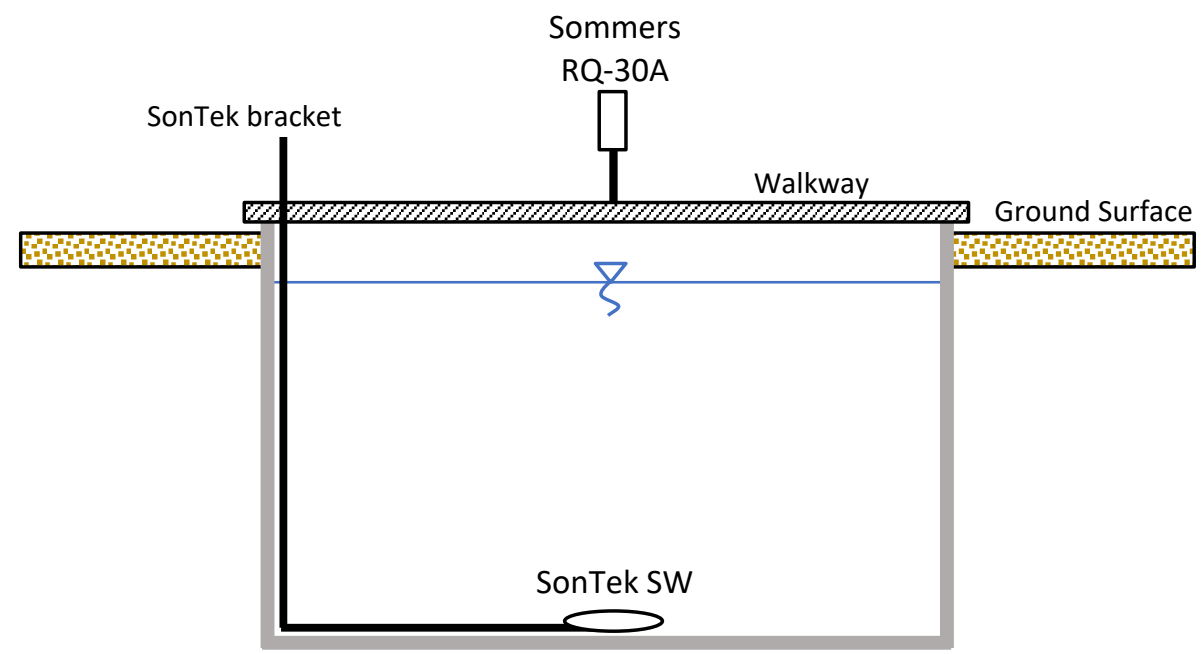

Figure 3. Cross section view of Installation \#1, looking downstream

The lift station just upstream of the field site is operated under manual flow control, with only negligible fluctuations in discharge throughout the day due to limited tidal influences affecting the pump TDH and discharge flow rate.

\section{Installation \#2 Overview}

About $300 \mathrm{ft}$ downstream of a different main canal lift pump station an ITRC subcritical contraction structure equipped with a SonTek SL acoustic doppler meter and a Sommer RQ-30A. The stationary discharge measurements were performed upstream of the structure. The following photos show the configuration of the sensors on the structure and the setup for the M9 boat discharge measurement. 


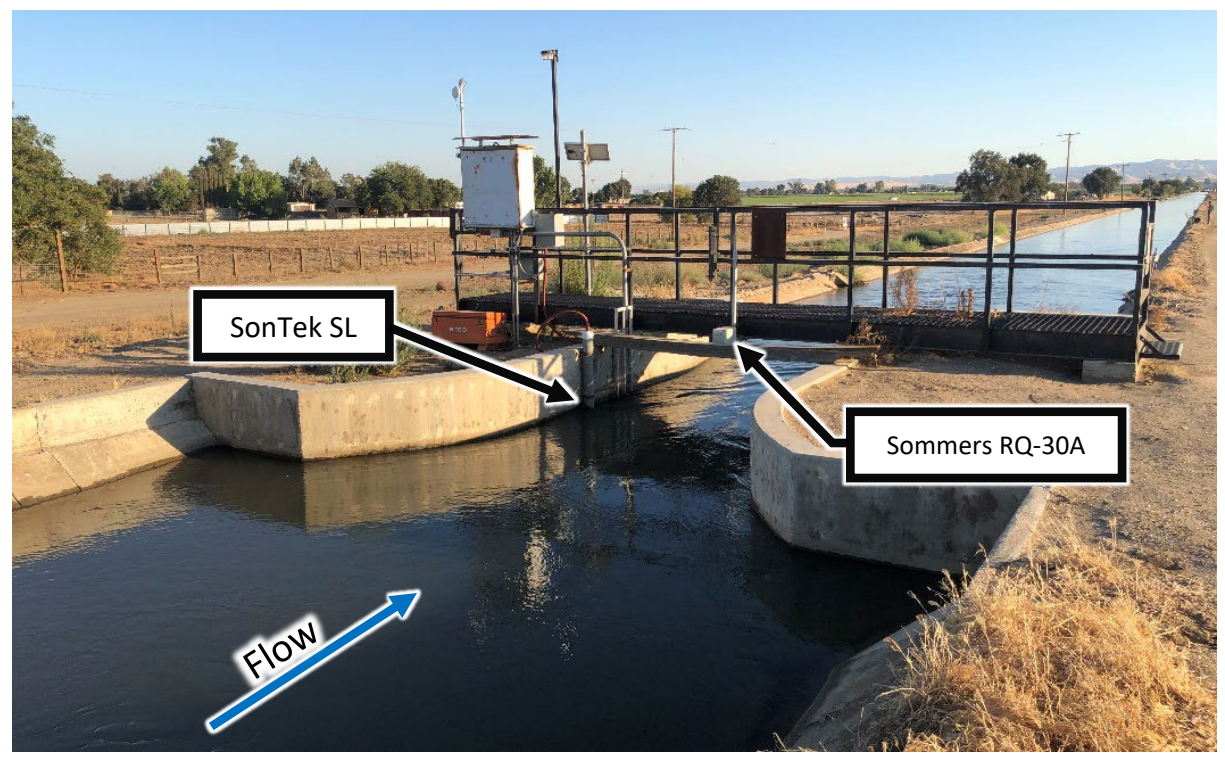

Figure 4. Installation \#2 layout

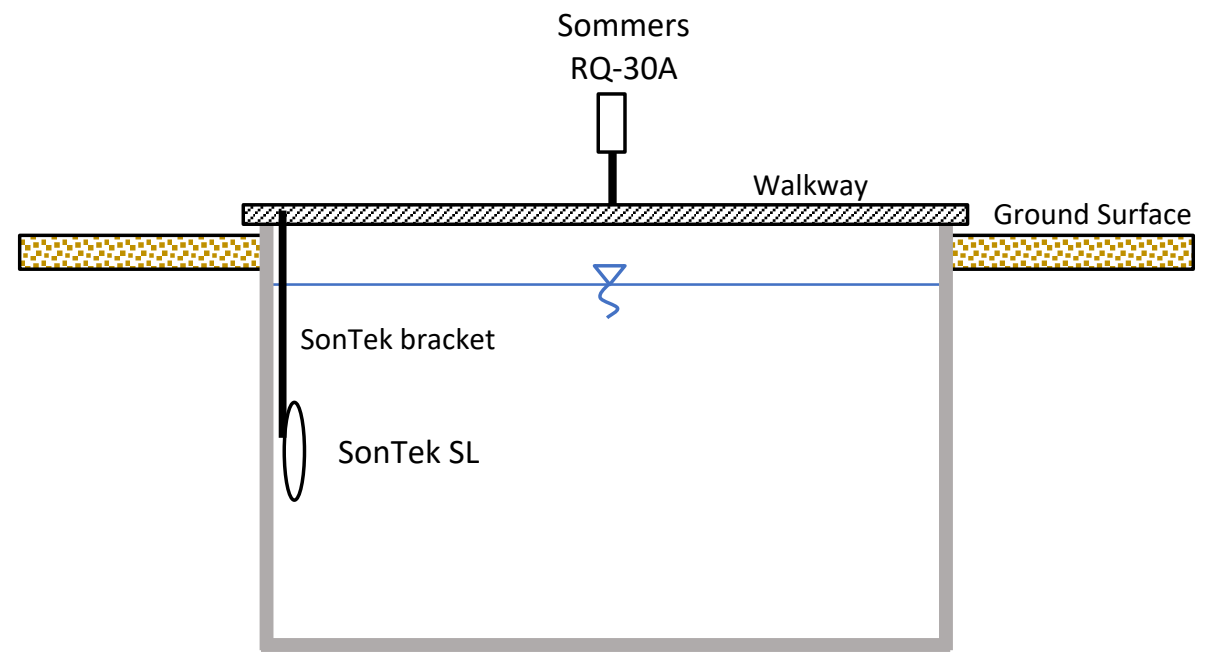

Figure 5. Cross section view of Installation \#2, looking downstream

The lift pump station just upstream of the Installation \#2 site is operated under automatic downstream control. Therefore, the pump discharge is constantly fluctuation to maintain a relatively constant downstream water level. To minimize main canal interruptions during the study, the pumps were left in automatic control throughout the study.

\section{RESULTS}

\section{Installation \#1 Discharge Measurements}

Table 2 compares the measured discharge of the SonTek M9 to the average observed measurements from the SonTek SW and Sommers RQ-30A units over the duration of the measurement. The results verified that both field devices are capable of excellent flow measurement accuracy. 
Table 2. Current metering data for Installation \#1

\begin{tabular}{|l|l|l|l|l|l|}
\hline \multirow{2}{*}{ Site Visit Date } & SonTek M9 & \multicolumn{3}{|l|}{ SonTek SW } & \multicolumn{2}{l|}{ Sommer RQ-30A } \\
\cline { 3 - 6 } & Measured (CFS) & Measured (CFS) & Error (\%) & Measured (CFS) & Error (\%) \\
\hline $4 / 17 / 2020$ & 142.4 & 140.7 & $-1.2 \%$ & 143.0 & $+0.4 \%$ \\
\hline $7 / 24 / 2020$ & 173.9 & 178.5 & $+2.6 \%$ & 167.4 & $-3.7 \%$ \\
\hline $8 / 21 / 2020$ & 96.7 & 98.0 & $+1.4 \%$ & 95.3 & $-1.5 \%$ \\
\hline Average Field Device Error (\%) & $+0.9 \%$ & $-1.60 \%$ & \\
\hline
\end{tabular}

\section{Installation \#1 Data Trends}

Data collected at Installation \#1 is shown in Figure 6. The average absolute percent difference between the SonTek SW and the RQ-30a for the dataset is 5.2\%. The standard deviation of the absolute error is about $4 \%$.

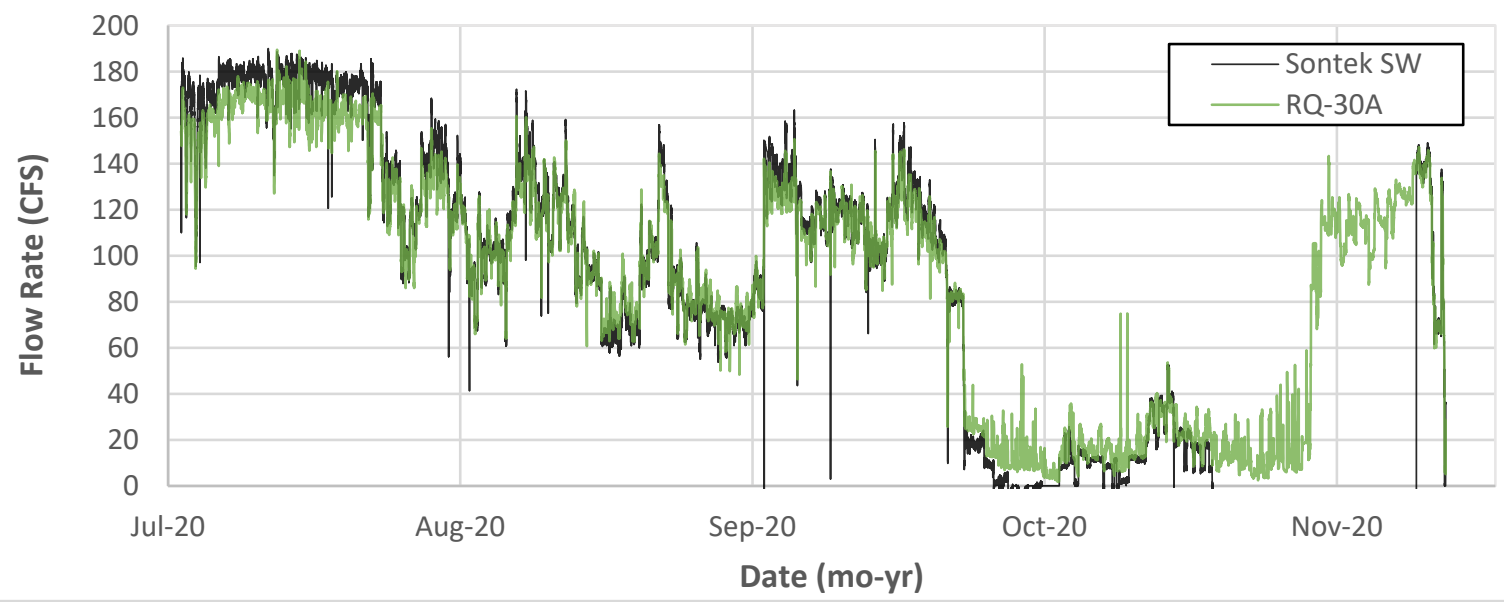

Figure 6. Data collected at Installation \#1 on approximately 15-minute intervals

\section{Installation \#2 Measurements}

Table 3 compares the measured discharge of the SonTek M9 to the average observed measurements from the SonTek SW and Sommers RQ-30A units.

Table 3. Current metering data for Installation \#2

\begin{tabular}{|l|l|l|l|l|l|}
\hline \multirow{2}{*}{ Site Visit Date } & SonTek M9 & SonTek SL & \multicolumn{2}{l|}{ Sommer RQ-30A } \\
\cline { 3 - 6 } & Measured (CFS) & Measured (CFS) & Error (\%) & Measured (CFS) & Error (\%) \\
\hline $7 / 25 / 2019$ & 100.9 & 103.9 & $+2.9 \%$ & 126.4 & $+25.3 \%$ \\
\hline $4 / 16 / 2020$ & 101.6 & 101.4 & $-0.1 \%$ & 116.7 & $+14.9 \%$ \\
\hline $7 / 23 / 2020$ & 112.1 & 116.6 & $+4.1 \%$ & NO DATA \\
\hline $8 / 21 / 2020$ & 121.1 & 110.3 & $-8.9 \%$ & 128.0 & $+5.6 \%$ \\
\hline $10 / 16 / 2020$ & 91.8 & 91.6 & $-0.2 \%$ & 119.0 & $+29.6 \%$ \\
\hline Average Field Device Error (\%) & $-0.5 \%$ & $+18.9 \%$ & \\
\hline
\end{tabular}


The results verified that both field devices have notably more variability in flow measurement accuracy compared to Installation \#1, without self-evident causes.

\section{Installation \#2 Data Trends}

A data trend for Installation \#2 is shown in Figure 7. As shown in the graph, the RQ-30 and SonTek SL tend to track together as canal flows fluctuate. A histogram was developed for the data to better illustrate the frequency and magnitude of error over the entire time series.

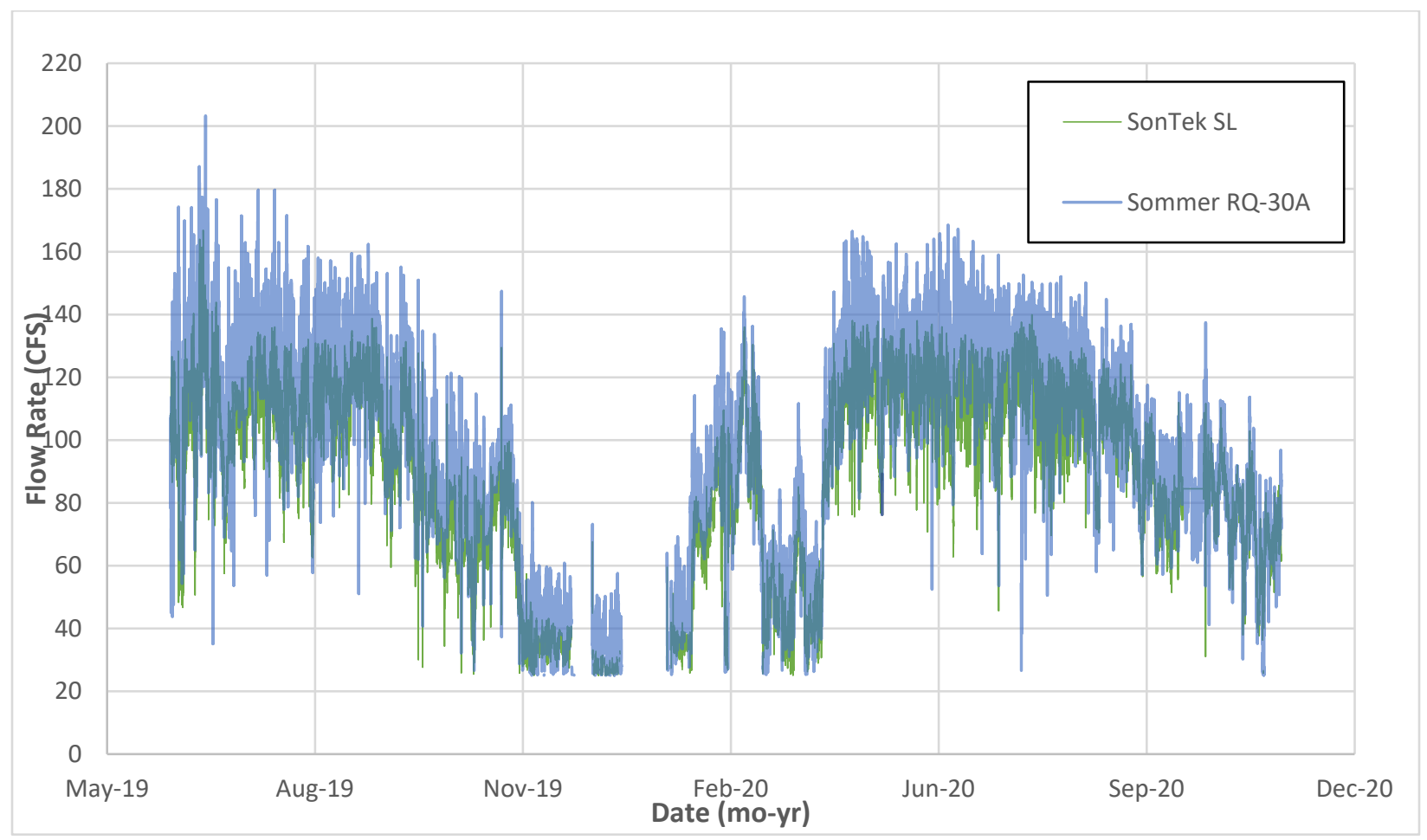

Figure 7. Installation \#2 time series plot showing measured SonTek SL and Sommer RQ-30A data using approximately 1 hour time intervals between data points

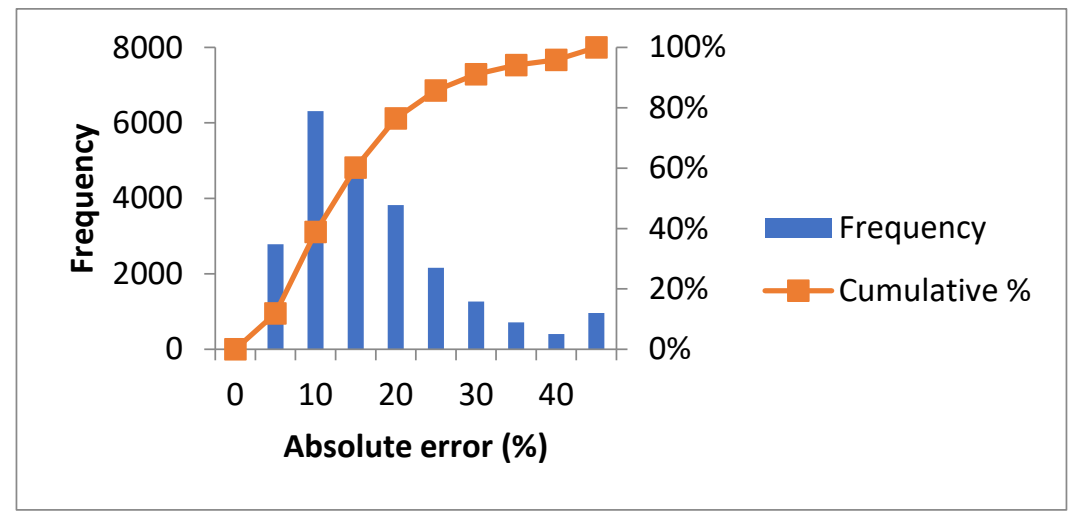

Figure 8. Histogram chart of Installation \#2 data

The chart indicates that while the about $80 \%$ of the non-contact meter data points exhibit $17 \%$ or less difference from the SonTek SL. 


\section{DISCUSSION}

The study results show significant performance differences between two nearly identically configured sites. Each site features an existing ADVM device operating in parallel with the installed RQ-30A at a hydraulic conditioning structure. The Installation \#1 data for both the preexisting SonTek SW and RQ-30A is considered excellent by the authors at an average error of about $5 \%$ or less.

On the other hand, the performance at Installation \#2 is variable. For example, the error between the SonTek M9 RiverSurveyor and the SonTek SL range from -9\% to about 4\%. Similarly, the error between the SonTek M9 and the RQ-30A varies between about 5\% to about $30 \%$. This indicates that a simple linear regression calibration would be unable to calibrate either device.

\section{CONCLUSION}

The data indicates the following:

- Non-contact meters can be successfully deployed for flow measurement in irrigation open channels .

- It is critical to field verify/calibrate any flow measurement device rather than just assume its error is acceptable "out-of-the-box."

- Non-contact meters can be considered by irrigation engineers as a viable alternative to ADVMs or as a redundant flow measurement device where continuous monitoring and public reporting of diversion flows is mandated by regulatory agencies. 